\title{
The impact, among third-level students, of nutrition knowledge on behaviour towards products with health claims
}

\author{
F. Lalor, J. Kennedy and P. Wall \\ National Nutrition Surveillance Centre, School of Public Health and Population Science, University College Dublin, \\ Dublin 4, Republic of Ireland
}

Over the last 10 years functional foods (foods containing nutrients with health-enhancing properties over and above those of conventional foods) have appeared on the marketplace and have the potential to improve health by improving diet. A variety of studies have demonstrated varying extents of support for the belief that nutritional knowledge has an influence on dietary behaviour ${ }^{(1-3)}$. The current study investigates whether nutritional knowledge has an impact on the credibility of health claims made on functional foods or on the purchasing behaviour of functional foods.

A web-based survey, targeting third-level students (students attending universities and colleges of higher education), was conducted in March 2008 and 226 completed questionnaires were included for analysis. Participants' level of nutrition knowledge was measured using a modified version of the general nutrition knowledge questionnaire ${ }^{(4)}$ and perceived credibility was gauged using a seven-point semantic differential scale. Participants' purchasing behaviour of functional foods was also assessed on a seven-point scale.

Females scored significantly higher than males for nutrition knowledge $(P=0.004)$ and some significant results across different carrier products (breakfast cereal, milk, yoghurt and cheese) suggest a trend whereby an increase in nutrition knowledge may lead to a decrease in perceived credibility of certain health claims. The more established claim 'Calcium for healthy bones and teeth' received the highest credibility score $(76.2 \%)$ compared with 'Boosted with probiotic cultures for healthy digestion' (53\%), 'Fortified with fibre to reduce cholesterol' (45\%) and 'Boosted with plant stanols which can help lower cholesterol' $(45 \%)$. When claims were linked with specific carrier products the credibility ratings began to fall. There was no significant difference between the purchasing behaviour of those with a high or low level of nutrition knowledge.

A higher level of nutrition knowledge leads to a lower level of credibility of health claims. Level of nutritional knowledge does not impact on purchasing behaviour of products with health claims. Information alone is not enough.

1. Kristal AR, Bowen DJ, Curry SJ et al. (1990) Health Educ Res 5, 467-477.

2. Patterson C, Kristal A, Lynch J et al. (1995) J Nutr Educ 27, 86-92.

3. Frewer L, Scholderer J \& Lambert N (2003) Br Food J 105, 714-731.

4. Parmenter K \& Wardle J (1999) Eur J Clin Nutr 53, 298-308. 